\title{
Diagnostic Accuracy of Dermoscopy for Scabies
}

\author{
Feng-Zeng Li* $\odot$, Shuang Chen \\ Department of Dermatology, The First Affiliated Hospital of Chongqing Medical University, Chongqing 400016, China
}

\begin{abstract}
The diagnostic accuracy of dermoscopy (DS) for scabies, a highly contagious parasitic disease, remains disputed. This study aimed to assess the diagnostic accuracy of DS in scabies, analyze the factors influencing DS, and explore its role in post-treatment evaluation. Patients with suspected scabies were randomly divided into 2 groups: 71 patients in the skin scraping (SS) group and 73 patients in the DS group. The diagnostic efficiencies of SS and DS in these groups were calculated. We also analyzed the influence of body part and investigator competence on the accuracy of DS. Then 16 body parts with typical signs of scabies were monitored by DS 2 and 4 day after sulfur ointment treatment. The sensitivity and specificity of DS were $98.3 \%$ and $88.5 \%$, respectively. Hands, arms, and the abdomen had higher positivity rates than other body parts $(P<0.001)$. The accuracy of dermatologists' interpretations of images negative for scabies in the intermediate- and high-level groups was higher than that in the low-level group $(P<0.001)$. At follow-up, the mites were still visible on $43.8 \%$ to $62.5 \%$ of the skin lesions 2 and 4 day after sulfur ointment treatment. These results showed that DS could significantly increase the accuracy of diagnosing scabies owing to its high sensitivity and specificity. Therefore, it may be useful for monitoring clinical responses to anti-parasitic treatment.
\end{abstract}

Key words: Scabies, dermoscopy, diagnosis, accuracy

\section{INTRODUCTION}

Scabies, a common skin disease that affects more than 200 million people globally, was considered a neglected tropical disease by the World Health Organization in 2017 [1]. This infectious disease caused by S. scabiei var. hominis, which spreads easily through direct skin-to-skin contact and usually occurs in undeveloped, war-torn countries or regions [2]. However, scabies may still affect vulnerable populations in developed countries, such as students, aged people, or inpatients, leading to mini-epidemics in families, dormitories, or wards [3,4]. It usually presents with herpes, papulovesicles, nodules, and intense pruritus at night, which cause pain and disturbed sleep in the affected individuals. Accurate and prompt diagnosis of scabies, along with appropriate isolation and treatment, is crucial to preventing its spread [5]. At present, the diagnosis is primarily based on clinical manifestations and microscopic examination. Owing to the heterogeneity of clinical manifestations and false-negative results, this condition may easily be misdi-

\footnotetext{
- Received 19 November 2019, revised 27 September 2020, accepted 25 October 2020. *Corresponding author (sdlfz2008@126.com) (c) 2020, Korean Society for Parasitology and Tropical Medicine This is an Open Access article distributed under the terms of the Creative Commons Attribution Non-Commercial License (https://creativecommons.org/icenses/by-nc/4.0) which permits unrestricted non-commercial use, distribution, and reproduction in any medium, provided the original work is properly cited.
}

agnosed during a clinical assessment.

Dermoscopy (DS), a noninvasive optical magnifying technique, has been used to aid in the diagnosis of scabies in recent years $[6,7]$. However, its accuracy is still disputed [8-10]. In this study, we assessed the diagnostic accuracy of DS for scabies, analyzed the factors influencing DS, and examined its role in monitoring clinical responses to anti-parasitic treatment.

\section{MATERIALS AND METHODS}

\section{Ethicsstatement}

The study protocol was approved by the Medical Ethics Committee of the First Affiliated Hospital of Chongqing Medical University. Ethical approval was given by the Medical Ethics Committee, the First Affiliated Hospital of Chongqing Medical University, and an approval was given under reference number 2017-127. Written informed consent was obtained from all study participants. All procedures were performed in accordance with the Declaration of Helsinki and relevant policies in China.

\section{Patients}

All patients suspected of having scabies based on clinical symptoms in the Department of Dermatology of the First Af- 
filiated Hospital of Chongqing Medical University were prospectively enrolled in the study from June 10, 2017, to March 15, 2019 .

\section{Study design}

Firstly, a dermatologist evaluated patients with a suspicion of scabies and recorded clinical data including sex, age, symptoms, and the location and characteristics of skin lesions (papules, vesicles, pustules, burrows, plaques, nodules, and severe itching). A total of 144 patients were included in the study and randomly divided into 2 groups: 71 patients in the skin scraping (SS) group and 73 patients in the DS group. There were no statistical differences in clinical features between the 2 groups (Table 1). Patients in the SS group were firstly referred to an investigator for SS examinations and then referred to another investigator for DS examinations. Patients in the DS group underwent the 2 diagnostic procedures in reverse order. When there was a discrepancy (negative SS finding/ positive DS finding), an SS examination was performed on the positive site, guided by DS. Supplementary Fig. S1 presents a flow chart of the protocol for this study. Additionally, the relationships between the locations of lesions and positive results of DS were recorded.

The influences of severity and duration of scabies on the sensitivity of DS were also investigated. Patients in the DS group who were diagnosed with scabies were subdivided into 3 severity groups (mild, moderate, and severe) based on the number of body parts involved and the types of lesions and another 3 groups (short, intermediate, and long) based on the duration of infestation, with 4 and 8 week as the cutoff points [10]. The categories are shown in Supplementary Table S1.

To investigate the influence of investigator competence on the accuracy of DS, we mixed 50 positive images ( 25 images with a typical "jet with contrail" sign and a clean background, 25 images with a busy background) and 30 negative images from patients with dermatitis, eczema, prurigo, and urticaria papulosa. All of these images were interpreted by 9 investigators with different skill sets and experience levels in DS. These investigators included 3 dermatologists with a preliminary understanding of the dermoscopic characteristics of scabies, 3 dermatologists with brief professional training in DS, and 3 dermatologists who had received professional training and been engaged in DS for more than 1 year. Additionally, some patients who were diagnosed with scabies by DS underwent DS detection on the same positive body parts 2 and 4 day after treatment to observe changes in the images.

\section{SS examination}

The skin lesions of patients suspected of having scabies were disinfected with $75.0 \%$ ethanol solution. Non-excoriated and

Table 1. Clinical features of patients suspected with scabies

\begin{tabular}{|c|c|c|c|}
\hline & SS group $(n=71)$ No. $(\%)$ & DS group ( $n=73$ ) No. (\%) & Total $(n=144)$ No. $(\%)$ \\
\hline \multicolumn{4}{|l|}{ Demographics } \\
\hline Male & $45(63.4)$ & 43 (58.9) & $88(61.1)$ \\
\hline Female & $26(36.6)$ & $30(41.1)$ & $56(38.9)$ \\
\hline Age range & $7-63 \mathrm{yr}$ & 6 month-69 yr & 6 month-69 yr \\
\hline Mean age (yr) & $40.4 \pm 12.3$ & $36.8 \pm 15.8$ & $38.2 \pm 13.6$ \\
\hline \multicolumn{4}{|l|}{ Symptoms and signs } \\
\hline Papulovesicles & $54(76.1)$ & $58(79.5)$ & $112(77.8)$ \\
\hline Vesicles & $38(53.5)$ & $46(63.0)$ & $84(58.3)$ \\
\hline Erythema\&scale & $60(84.5)$ & 65 (89.0) & $125(86.8)$ \\
\hline Scrotum nodules & 15 (33.3\% of men) & 18 (41.9\% of men) & 33 (37.5\% of men) \\
\hline Itch & $71(100)$ & $73(100)$ & $144(100)$ \\
\hline \multicolumn{4}{|l|}{ Body parts ${ }^{a}$} \\
\hline Hands & $43 / 71(60.6)$ & $50 / 73(68.5)$ & $93 / 144(64.6)$ \\
\hline Arms & $19 / 47(40.4)$ & $16 / 45(35.6)$ & 35/92 (38.0) \\
\hline Abdomen & 11/34 (32.4) & 15/39 (38.5) & 26/73 (35.6) \\
\hline Thighs & $5 / 22(22.7)$ & 6/19 (31.6) & $11 / 41(26.8)$ \\
\hline Scrotum & $7 / 22(31.8)$ & $8 / 25(32.0)$ & $15 / 47(31.9)$ \\
\hline Others & 3/13 (23.1) & 4/16 (25.0) & $7 / 29(24.1)$ \\
\hline
\end{tabular}

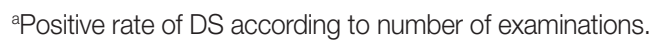

SS, skin scraping; DS, dermoscopy. 
non-inflamed areas were selected for SS. A drop of mineral oil was placed on the rash, and the scraped material was transferred to a slide and covered with a coverslip. Two specimens were obtained from each skin lesion. Reading was performed within 20 min at $100 \times$ and $400 \times$ magnification. Generally, such a mineral oil examination is regarded as the gold standard for diagnosing scabies. Skilled technicians are expected to find the bodies of mites, stumps, and eggs using optical microscopy to confirm the diagnosis of scabies.

\section{SS examination guided by DS}

A small mark was made next to the "jet with contrail" sign on the positive skin lesions under DS. SS was performed on the marked skin lesions, as described above. DS was performed once more to check whether the "jet," corresponding to a mite, was scraped down.

\section{DS examination}

Dermoscopy (Dermat Image System; Dermat, Beijing, China) was performed in our Department of Dermatology by examining physicians who had received specialized training from the Chinese National Telemedicine and Connected Health Center. DS was performed at $20 \times$ magnification for the initial screening and followed at $50 \times$ magnification to confirm the presence of mites. Whenever the "jet with contrail" sign was visible, a positive dermoscopic diagnosis of scabies was established. Furthermore, the presence of burrows in the skin and droppings in the tunnels at more than $20 \times$ magnification provided indirect evidence for the diagnosis of scabies.

\section{Final diagnosis of scabies}

A diagnosis of scabies was established by the presence of mites or eggs under an optical microscope.

\section{Data analysis}

The chi-square test was used to analyze data, and a $P$-value less than 0.05 was considered statistically significant.

\section{RESULTS}

\section{Diagnostic properties of SS and DS}

Of the 144 patients, 118 were diagnosed with scabies, based

Table 2. Diagnostic properties of dermoscopy and skin scraping for scabies

\begin{tabular}{lccc}
\hline Diagnostic property & Skin scraping (\%) & Dermoscopy (\%) & $P$-value \\
\hline Sensitivity (TP/TP+FN) & $51.7(61 / 118)$ & $98.3(116 / 118)$ & 0.000 \\
Specificity (TN/TN+FP) & $100.0(26 / 26)$ & $88.5(23 / 26)$ & 0.234 \\
Negative predictive value (TN/TN+FN) & $31.3(26 / 83)$ & $92.0(23 / 25)$ & 0.000 \\
Positive predictive value (TP/TP+FP) & $100(61 / 61)$ & $97.5(116 / 119)$ & 0.525 \\
\hline
\end{tabular}

FN, false negative; TN, true negative; TP, true positive; FP, false positive.

A

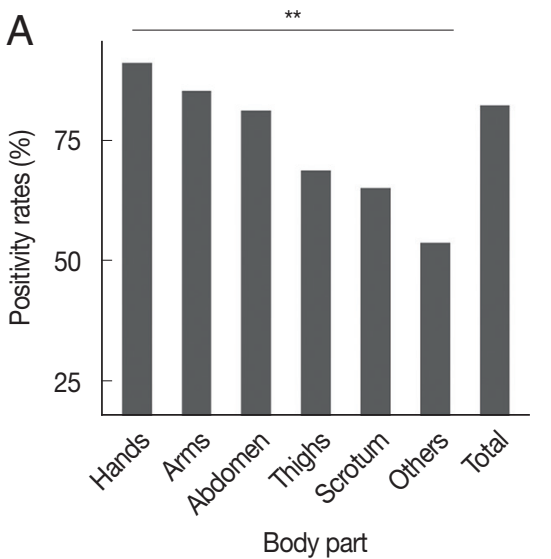

B

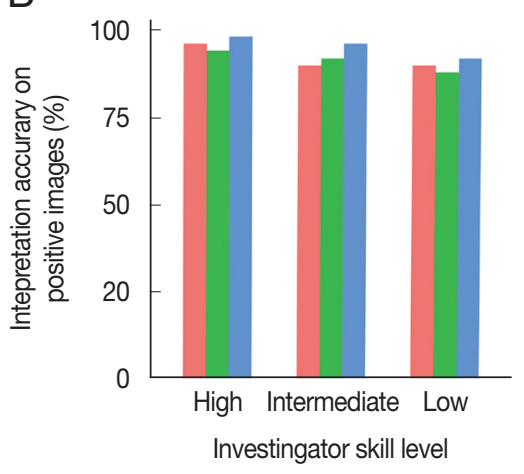

C

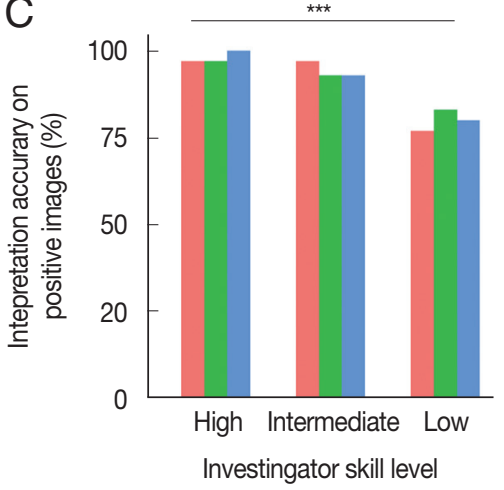

Fig. 1. Influence of body part and investigator skill level on the accuracy of dermoscopy. (A) Difference in positive rate of dermoscopy among different body parts $\left(X^{2}=19.723, P<0.01\right)$. (B) Interpretation accuracy on positive images. No difference in accuracy was observed among the 3 groups $\left(X^{2}=4.104, P>0.05\right)$. (C) Interpretation accuracy on negative images. Interpretation accuracy on the lowlevel group was lower than that on the intermediate-level and high-level groups $\left(X^{2}=19.131, P<0.001\right)$. 
on the presence of mites or eggs as seen under an optical microscope. The diagnostic properties of SS and DS are summarized in Table 2. The sensitivity of DS was significantly higher than that of SS $\left(98.3 \%\right.$ vs. $\left.51.7 \%, \chi^{2}=68.362, P<0.001\right)$. DS and SS had a similar specificity $\left(88.5 \%\right.$ vs. $\left.100.0 \%, \chi^{2}=1.415, P=0.234\right)$.

\section{Positive rate for different body parts using DS}

DS revealed positive signs in 187 of 227 different body parts of the patients with scabies. As shown in Fig. 1A, positivity rates differed significantly by body part $\left(\chi^{2}=19.723, P=0.001\right)$. Hands $(93 / 102,91.2 \%)$ showed the highest positivity rate, followed by arms (35/41, 85.4\%), the abdomen $(26 / 32,81.3 \%)$, thighs (11/16, 68.8\%), the scrotum in male patients $(15 / 23,65$. $2 \%)$, and other parts $(7 / 13,53.8 \%)$ including the chest, back, and feet.

\section{Sensitivity of DS according to severity and duration of scabies}

The 63 patients in the DS group diagnosed with scabies were divided into mild ( $\mathrm{n}=34)$, moderate $(\mathrm{n}=26)$, and severe $(\mathrm{n}=3)$ scabies groups (Supplementary Table $\mathrm{S} 1)$. The sensitivity of these 3 groups was $94.1 \%$ (32/34), 100.0\% (26/26), and
$100.0 \%$ (3/3), respectively. No significant difference was observed among these groups $\left(\chi^{2}=1.662, P=0.436\right)$.

These patients were also divided into short $(n=41)$, intermediate $(\mathrm{n}=15)$, and long $(\mathrm{n}=7)$ groups based on the duration of their scabies (Supplementary Table S1). The sensitivity of these 3 groups was $100.0 \%$ (41/41), 93.3\% (14/15), and $85.7 \%$ (6/7), respectively. No significant difference was observed among these groups $\left(\chi^{2}=4.751, P=0.093\right)$.

\section{Accuracy of investigators with different skills and experience levels in interpreting DS images}

A total of 80 images, including 50 images with positive signs and 30 images without positive signs, were interpreted in a blind manner by 9 dermatologists who were divided into 3 groups, low- $(\mathrm{n}=3)$, intermediate- $(\mathrm{n}=3)$ and high-level $(\mathrm{n}=3)$ groups, based on their skill and experience in DS. As shown in Fig. 1B, the accuracy of interpreting the positive images was similar among the 3 groups $\left(\chi^{2}=4.104, P=0.129\right)$. The accuracy of interpreting the negative images was higher in the intermediate- and high-level groups than in the low-level group $\left(\chi^{2}=19.131, P<0.001\right)$ (Fig. 1C).
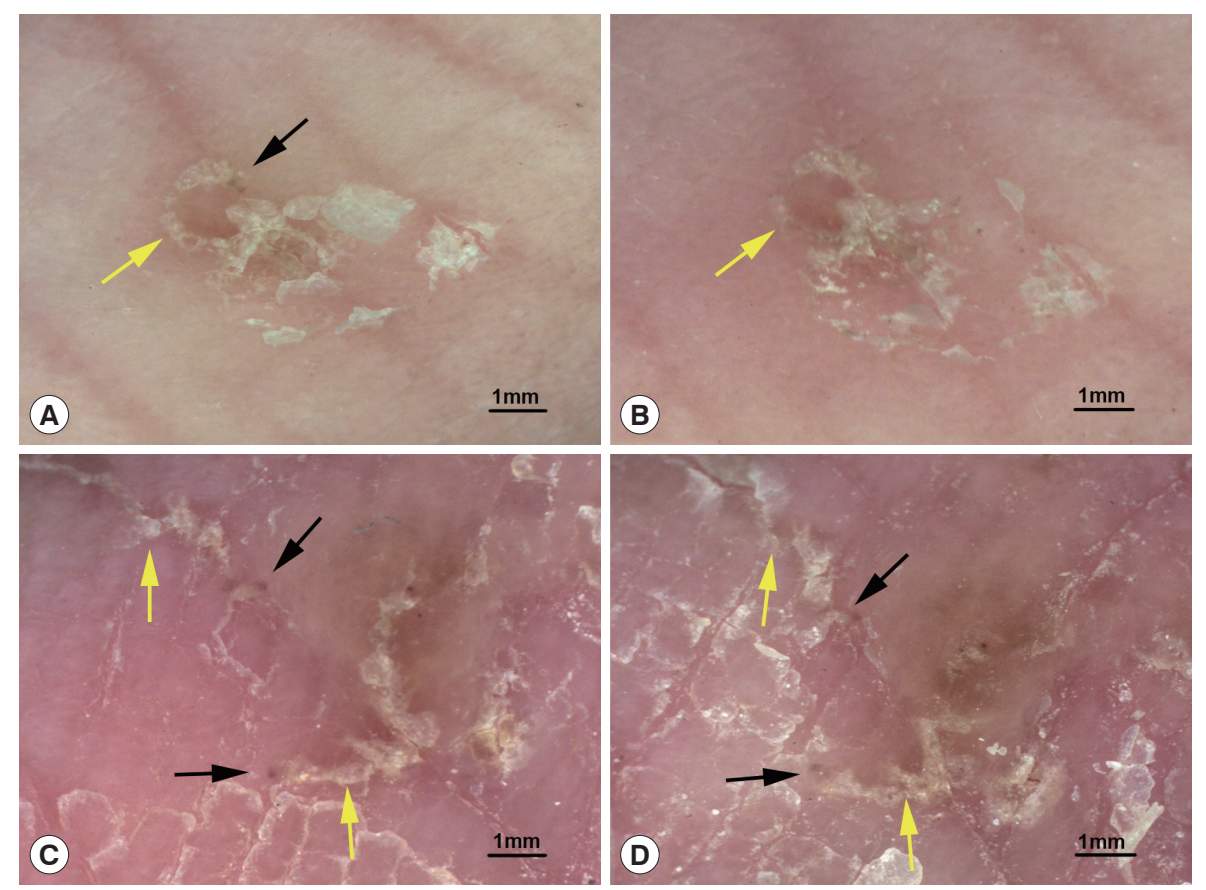

Fig. 2. Typical structures of mites and burrows before and after treatment. (A) A mite (black arrow) and a burrow (yellow arrow) before treatment. (B) 2 day after sulfur ointment treatment, the mite has disappeared, and the burrow is slightly damaged. (C) Mites (black arrow) and burrows (yellow arrow) before treatment. (D) 4 day after sulfur ointment treatment, fuzzy mites and burrows are still visible (dermoscopic images at $20 \times$ magnification). 


\section{Dermoscopic features of scabies in post-treatment follow-up}

A total of 16 body parts of patients showing the typical structure of mites and burrows before treatment were selected for DS detection 2 and 4 day after treatment with sulfur ointment. On the 2nd day after treatment, the mites (triangular brown structures) were visible in 10 body parts (62.5\%, 10/ 16), and the burrows (curved white lines) were slightly damaged. On the 4th day after treatment, mites were visible in only 7 body parts $(43.8 \%, 7 / 16)$ and had become less clear, and the burrows were subject to different degrees of damage. Fig. 2 shows the changes in typical structures of mites and burrows before and after treatment.

\section{DISCUSSION}

DS is a non-invasive technique that magnifies the skin for observation in vivo. Morphologic features, otherwise invisible to the naked eye, are displayed on a computer screen. DS is usually performed with a polarized light source or by the application of an immersion liquid (oil or water) to the skin, which helps to eliminate light reflection [11]. Pigmented structures and the vasculature of the skin surface to the superficial dermis can be inspected using DS; therefore, it is effective for identifying scabies mites and burrows in vivo [5-7,11-13].

However, the diagnostic accuracy of DS for scabies remains disputed. A study of 100 patients compared the diagnostic properties of SS, adhesive tape, and DS [8]. The sensitivity of both DS and SS were 43.5\%, lower than that of the adhesive tape (69.6\%). Furthermore, the specificity of DS (84.4\%) was lower than that of SS (100.0\%) and the adhesive tape (100.0\%) [8]. In 2 other studies, the sensitivity of DS was $83.0 \%$ [10] and $95.0 \%$ [9], higher than that of DS in the above study. The specificity of DS in the study by Walter et al. [10] was only $46.0 \%$, much lower than the specificity $(86.0 \%)$ observed by Dupuy et al. [14]. In the present study, the sensitivity of SS was $51.7 \%$ (61/118), and the sensitivity and specificity of DS were $98.3 \%$ (116/118) and 88.5\% (23/26), respectively. The sensitivity of DS was much higher than that of SS $(P<0.001)$, and the specificity of DS was similar to that of SS $(P=0.234)$, which indicates that DS has high diagnostic accuracy for scabies.

The sensitivity and specificity of DS may be influenced by the body part. Mites may be undetectable when on dark skin or areas with hair, dirt, or tiny blood spots [8]. In this study, positive signs were noted on 187 of 227 different body parts. The sensitivity of DS for different body parts ranged from $56.5 \%$ to $91.2 \%$. Hands, arms, and the abdomen had the highest positivity rates for mites $(P<0.001)$. These areas with tender skin are the most pre-disposed to infection by scabies mites and should be prioritized for detection to improve the positivity rates [5].

The sensitivity of DS increased with the severity of scabies but decreased with the duration of the infestation. Similar results were reported by Walter et al. [10]. However, the sensitivity difference of DS in patients with varying severity or duration was not significant in this study $(P>0.05)$, which may be because of the near-perfect sensitivity of DS (up to 98.3\%) and the small sample size. Moreover, in clinical practice, the severity of scabies is not usually assessed, except for Norwegian scabies and bullous scabies, because most patients present with mild clinical symptoms and a short duration.

We also evaluated the interpretational accuracy of dermoscopic images among 9 dermatologists who were divided into 3 groups based on their skill level. No differences were observed in the interpretation of positive images among the 3 groups. However, there were differences among the 3 groups in the interpretation of negative images. The low-level group showed lower accuracy than intermediate-level and high-level groups $(P<0.001)$. The results showed that typical positive signs of scabies are simple and easily identifiable in DS images, while there may be some difficulties for beginners in the elimination of interference factors and accurate identification of negative images, which may be overcome by professional training and practice. Thus, DS shows high sensitivity and low specificity even when performed by beginners and could be used as a screening test.

Additionally, recommended treatments for scabies include permethrin 5\% cream, benzyl benzoate 25\% lotion, and sulfur $6-33 \%$ ointment [15]. Unfortunately, permethrin cream is unavailable in China, and sulfur ointment remains the most commonly used treatment. In the post-treatment follow-up, patients with a "jet-contrail" sign underwent dermoscopic detections 2 and 4 day after sulfur ointment treatment. Mites were still visible on $43.8 \%$ (7/16) to $62.5 \%$ (10/16) of the skin lesions. We speculated that the mites crawled away or fell off the body after their death, and the visible mites died as a result of the treatment. A decrease in the number of mites without the appearance of new skin lesions indicates a good therapeutic effect. DS detection enhances the monitoring of clinical re- 
sponse to treatment in scabies, which may help minimize the risk of overtreatment, reduce potential side effects, and enhance patient compliance [16]. Furthermore, sulfur ointment is not the best treatment option for scabies, and infection may recur in some patients even after treatment. Systematic treatment monitoring with DS and SS may yield much better results.

Our study suggests that DS may significantly increase the accuracy of diagnosing scabies owing to its sensitivity and specificity. The priority selection of lesions on the hands, arms, and the abdomen for detection and screening by professionally skilled investigators can help achieve an accurate diagnosis of scabies. DS may also help in monitoring the clinical responses to anti-parasitic treatment and detecting the recurrence or reinfection of scabies.

\section{ACKNOWLEDGMENTS}

This study was supported by a grant from the First Affiliated Hospital of Chongqing Medical University (No. PYJJ2017-22).

\section{CONFLICT OF INTEREST}

The authors declare no conflict of interest related to this study.

\section{REFERENCES}

1. World Health Organization. Neglected tropical diseases: Scabies and other ectoparasites [Internet]; [cited 2020 August 5]. Available from: https://www.who.int/neglected_diseases/diseases/ scabies-and-other-ectoparasites/en/

2. Mueller SM, Gysin S, Schweitzer M, Schwegler S, Haeusermann P, Itin P, Bart T, Denz RS, Steffen T, Kuehl R, Widmer AF, Brandt O. Implementation and evaluation of an algorithm for the management of scabies outbreaks. BMC Infect Dis 2019; 19: 200.

3. Petit A, Bourrat E, Dehen L, Dupuy A. Scabies outbreaks in care homes for the elderly. Lancet Infect Dis 2018; 18: 1310. https:// doi.org/10.1016/S1473-3099(18)30665-0

4. Vijayan V, Marrero E, Gaspar A, Wisdom C, Honeycutt MD, Linam WM. Outbreak of scabies in a neonatal intensive care unit. Infect Control Hosp Epidemiol 2019; 40: 613-614. https:// doi.org/10.1017/ice.2019.57

5. Executive Committee of Guideline for the Diagnosis and Treat- ment of Scabies. Guideline for the diagnosis and treatment of scabies in Japan (third edition): Executive Committee of Guideline for the Diagnosis and Treatment of Scabies. J Dermatol 2017; 44: 991-1014. https://doi.org/10.1111/1346-8138.13896

6. Lallas A, Apalla Z, Lazaridou E, Sotiriou E, Vakirlis E, Ioannides D. Scabies escaping detection until dermoscopy was applied. Dermatol Pract Concept 2017; 7: 49-50. https://doi.org/10.5826/ dpc.0701a09

7. Micali G, Lacarrubba F, Verzi AE, Nasca MR. Low-cost equipment for diagnosis and management of endemic scabies outbreaks in underserved populations. Clin Infect Dis 2015; 60: 327-329. https://doi.org/10.1093/cid/ciu826

8. Abdel-Latif AA, Elshahed AR, Salama OA, Elsaie ML. Comparing the diagnostic properties of skin scraping, adhesive tape, and dermoscopy in diagnosing scabies. Acta Dermatovenerol Alp Pannonica Adriat 2018; 27: 75-78.

9. Cinotti E, Labeille B, Cambazard F, Biron AC, Chol C, Leclerq A, Jaffelin C, Perrot JL. Videodermoscopy compared to reflectance confocal microscopy for the diagnosis of scabies. J Eur Acad Dermatol Venereol 2016; 30: 1573-1577. https://doi.org/10.1111/ jdv.13676

10. Walter B, Heukelbach J, Fengler G, Worth C, Hengge U, Feldmeier $\mathrm{H}$. Comparison of dermoscopy, skin scraping, and the adhesive tape test for the diagnosis of scabies in a resource-poor setting. Arch Dermatol 2011; 147: 468-473. https://doi.org/10.1001/archdermatol.2011.51

11. Micali G, Lacarrubba F, Verzì AE, Chosidow O, Schwartz RA. Scabies: advances in noninvasive diagnosis. PLoS Negl Trop Dis 2016; 10: e0004691. https://doi.org/10.1371/journal.pntd.0004691

12. Tang J, You Z, Ran Y. Simple methods to enhance the diagnosis of scabies. J Am Acad Dermatol 2019; 80: 99-100. https://doi. org/10.1016/j.jaad.2017.07.038

13. Lacarrubba F, Ardigò M, Di Stefani A, Verzì AE, Micali G. Dermatoscopy and reflectance confocal microscopy correlations in nonmelanocytic disorders. Dermatol Clin 2018; 36: 487-501. https://doi.org/10.1016/j.det.2018.05.015

14. Dupuy A, Dehen L, Bourrat E, Lacroix C, Benderdouche M, Dubertret L, Morel P, Feuilhade de Chauvin M, Petit A. Accuracy of standard dermoscopy for diagnosing scabies. J Am Acad Dermatol 2007; 56: 53-62. https://doi.org/10.1016/j.jaad.2006.07.025

15. Salavastru CM, Chosidow O, Boffa MJ, Janier M, Tiplica GS. European guideline for the management of scabies. J Eur Acad Dermatol Venereol 2017; 31: 1248-1253. https://doi.org/10.1111/jdv. 14351

16. Micali G, Tedeschi A, West DP, Dinotta F, Lacarrubba F. The use of videodermatoscopy to monitor treatment of scabies and pediculosis. J Dermatolog Treat 2011; 22: 133-137. https://doi. org/10.3109/09546631003649687 